\title{
Where is the Lesion? Glomus Tumours of the Hand
}

\author{
Chris Yuk Kwan Tang, Timothy Tipoe, Boris Fung \\ Department of Orthopaedics and Traumatology, University of Hong Kong Medical Centre, Queen Mary Hospital, Hong Kong
}

Glomus tumours are vascular hamartomas that are commonly found in the hand, particularly the subungual region. They appear as solitary or multiple tumours, and often present as a bluish discoloration of the nail plate. Different diagnostic tests are outlined, as well as imaging studies such as magnetic resonance imaging and ultrasound. Misdiagnosis and delayed diagnosis of these tumours are common, while a familial tendency is a potential risk factor but not yet proven. Complete surgical excision often results in complete symptomatic relief, while recurrences are largely due to incomplete excision or the growth of a new glomus tumour. This article aims to review the key aspects of glomus tumours and provide a diagnostic algorithm so that the lesion can be recognized and treated earlier.

Keywords Glomus tumor / Disease / Hand

\author{
Correspondence: \\ Chris Yuk Kwan Tang \\ Department of Orthopaedics and \\ Traumatology, University of Hong \\ Kong Medical Centre, Queen Mary \\ Hospital, 5/F Professorial Block, \\ 102 Pokfulam Road, Hong Kong \\ Tel: $+852-22554654$ \\ Fax: +852-22554654 \\ E-mail: medic.chris.tang@gmail.com
}

No potential conflict of interest relevant to this article was reported.

\section{INTRODUCTION}

Glomus tumours are rare, often benign neoplasms arising from a neuromyoarterial structure called a glomus body that controls blood pressure and temperature by regulating blood flow in the cutaneous vasculature [1]. In particular, $1 \%$ to $4.5 \%$ of these special tumours present themselves in the hand [2]. In general, glomus tumours are most commonly seen in female patients in the subungual region in the hands, while men often experience these tumours in other body parts [3]. Within the hand, they are often found in the subungual regions, as well as in the nail matrix, nail bed, and pulp of a finger $[1,4]$. Some diagnostic tests for glomus tumour include the Love's pin test, Hildreth's test, and the cold sensitivity test [5]. Magnetic resonance imaging (MRI) is used in some cases to support clinical diagnosis [6] and in delineating the tumour's size and precise location. Glomus tumours are often misdiagnosed as other painful tumours such as neuroma or gouty arthritis because of the presence of pain [3]. This entity should be taken into account when doing differential diagnosis on suspected patients. Complete surgical excision often results in permanent symptomatic relief and a low incidence of recurrence [2].

Searches for English-language articles on glomus tumours were performed in the PubMed database. Aspects of epidemiology, clinical presentation, diagnosis, and management were reviewed.

\section{CLINICAL PRESENTATION AND TU- MOUR LOCATION}

Seventy-five percent of glomus tumours occur in the hand [7]. Of these, it is estimated that about $65 \%$ of glomus tumours present in the subungual region. In general, there are two types of glomus tumours, namely, solitary and multiple. Solitary glomus tumours are more common, while multiple glomus tumours rarely occur in the digits [3]. Solitary glomus tumours are mainly 
found in the extremities of the hand, such as the nail bed and in the subungual region [8]. Multiple glomus tumours have sometimes been found simultaneously with type 1 neurofibromatosis. These tumours are often painless, making them harder to diagnose correctly [3].

Glomus tumours are vascular, subcutaneous lesions. As such, they are often seen under the skin as a palpable mass [3]. Glomus tumours are often recognized as a bluish discoloration [9] or pinkish red spot [3] under a nail fold, while others increase the curvature of it. This is due to tumour multiplication under the skin, resulting in elevation of the nail fold [9]. Occasionally, a glomus tumour also causes nail deformity. If these clinical signs are present, a glomus tumour should be suspected and included in differential diagnosis.

\section{DIAGNOSIS}

Early recognition and diagnosis of glomus tumours is extremely important, as prolonged misdiagnosis or delayed diagnosis may lead to the exacerbation of a patient's symptoms. Clinical examination tests along with imaging support the correct diagnosis of these tumours.

The classical triad of glomus tumour symptoms includes severe pain, pinpoint pain, and cold sensitivity [10]. The tumour itself may not be obvious to the naked eye (Figs. 1,2). There are three main clinical tests that help in diagnosing glomus tumours. The first is Love's pin test, in which a pinhead is used to apply pressure to the suspected area. The point at which intense pain is registered confirms the affected area containing the glomus tumour. The second test is Hildreth's test. In this test, transient ischemia is induced along the arm by using a tourniquet. Owing

\section{Fig. 1. Glomus tumour not obvious preoperatively}

Left middle finger glomus tumour that is not obvious to the naked eye.

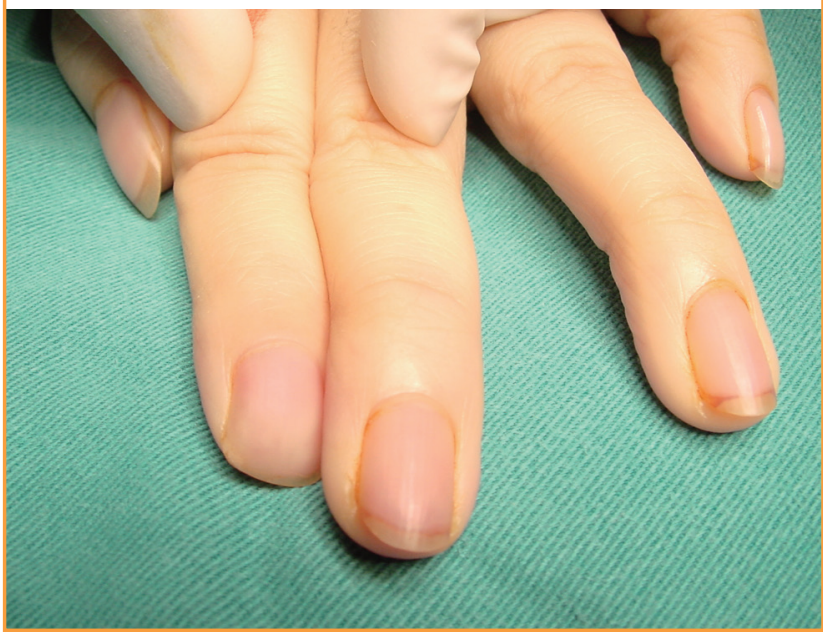

to the temporarily restricted blood supply, a positive test would be noted if the patient experiences a slight or full withdrawal of pain from the affected area. The reduction or absence of pain by repeating Love's pin test with the tourniquet also indicates a positive result. When the tourniquet is removed, the patient will feel a sudden return of pain. The third test is the cold-sensitivity test. In this test, cold water or an ice cube is applied to the affected area [5]. The patient experiencing increased pain would indicate a positive result.

Each of these clinical tests has different sensitivities, specificities, and accuracies. Netscher et al. [5] conducted an experiment on 18 patients to test the properties of each clinical examination. The results showed that the cold sensitivity test had $100 \%$ sensitivity, specificity, and accuracy. In comparison, Love's pin test had $100 \%$ sensitivity and $78 \%$ accuracy, while Hildreth's test was $71.4 \%$ sensitive, $100 \%$ specific, and $78 \%$ accurate. A combination of these tests should be used to increase the diagnostic yield.

There are other tests that have been used in diagnosing glomus tumours. One of these is the transillumination test, where light is passed through the finger pad in a darkened room [3]. The tumour region will show a red, opaque image, which helps in estimating its size. This test is $23 \%$ to $38 \%$ sensitive and $90 \%$ specific.

Imaging studies can also be performed in some cases where clinical examination does not support the diagnosis. MRI is used in detecting tumours equal or greater than $2 \mathrm{~mm}$ [5]. The characteristic image of glomus tumours on these scans is a high signal central dot surrounded by a zone of lower signal intensity [5]. They show low signal intensity on T1-weighted images, high signal intensity on T2-weighted images, and enhancement

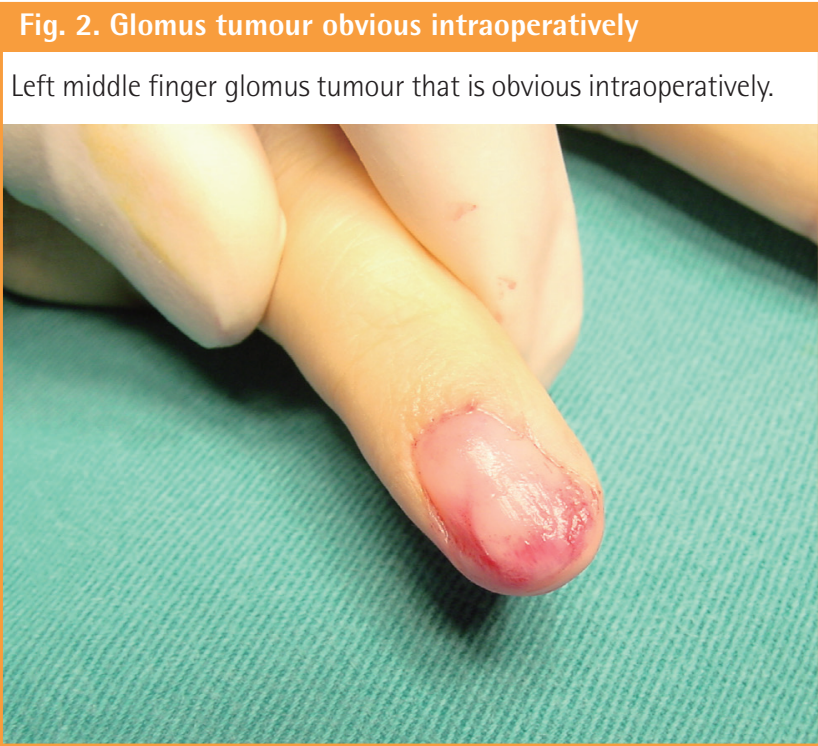




\section{Fig. 3. Diagnostic algorithm for glomus tumour}

$M R I$, magnetic resonance imaging.

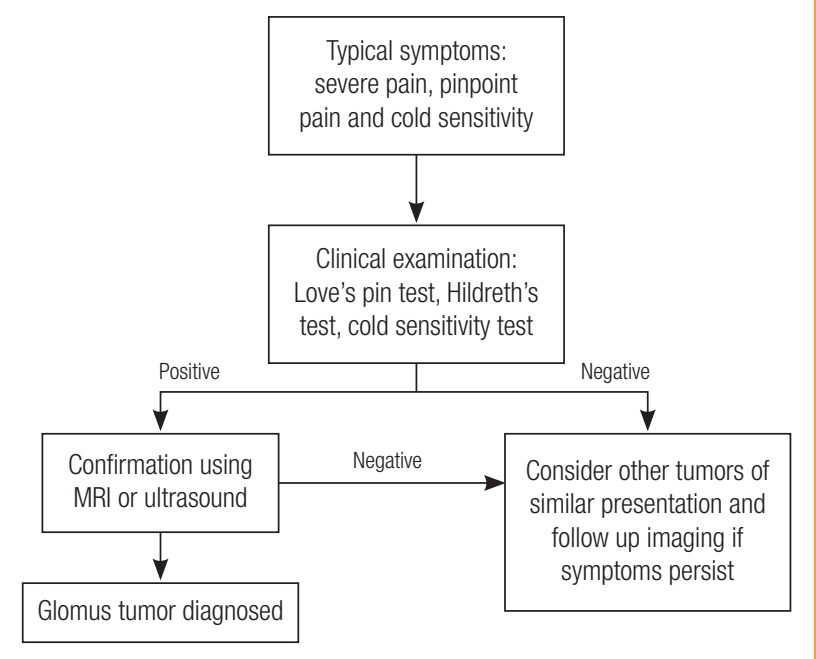

on T1-weighted images after gadolinium injection. MRI is also useful, as it delineates the size of the tumour and precise location, which increases the chance of complete excision. This also prevents overly extensive excision that may induce further unnecessary trauma on the patient and lead to postoperative nail deformity [6]. Ultrasound scanning images are also used in some cases for diagnosis of glomus tumours in the subungual region. This is done because it does not require preoperative excision of the tumour in any way and, unlike MRI, does not pose ionizing risks to the patient [11]. A simple diagnostic algorithm shown in Fig. 3 forms a basic guideline on the steps in diagnosing glomus tumours.

Glomus tumours are often misdiagnosed because the presence of pain on its own leads to suspecting other painful tumours. These include neuroma, gouty arthritis, etc. $[3,4,12,13]$. From the reviewed literature, it was found that delayed diagnosis ranged from 3 months to 15 years. Another reason for delay in diagnosis is that multiple glomus tumours tend not to be painful, while pain is a characteristic of most glomus tumours [3]. This makes differential diagnosis more difficult. Some of these tumours are also very deep in the skin, making them impalpable and thus more difficult to diagnose [14].

The reliability of MRI scans should also be taken into consideration. In a reviewed case report [13], 4 out of 40 patients (10\%) scanned using MRI had negative results, although histology confirmed them to have glomus tumours. This shows that although MRI is useful in the diagnosis of glomus tumours, it should only be done after a positive result is observed by clinical examination.

\section{MANAGEMENT}

The most common treatment for glomus tumours is surgical excision of the palpable mass. Complete surgical excision often results in complete symptomatic relief and a lessened risk of recurrence [2]. The use of MRI during diagnosis proves useful as it helps in defining the tumour better. This will hopefully lead to more accurate excision, also reducing the risk of recurrence and nail dystrophy [4]. Certain authors have reported that indomethacin can control the pain of glomus tumours for about 10 days [3].

Recurrence is reported by various authors to be $4 \%$ to $50 \%$ $[5,14]$. Subungual tumours are said to be more likely to recur [5], while skin-coloured glomus tumours increase the risk of recurrence because they are hard to delineate clearly. This increases the chance of incomplete excision and regrowth of the tumour [4]. In general, recurrences of the same glomus tumour are a result of incomplete excision, while other recurrences may be due to the growth of a new glomus tumour or pre-existing tumours already present at the time of surgery [1]. If symptoms of glomus tumours persist for more than 3 months, re-exploration of the affected area and repeat imaging should be done [5].

\section{CONCLUSIONS}

To conclude, although glomus tumours are uncommon tumours, symptoms and signs are relatively sensitive and specific when used in combination. Owing to its small size, diagnosis of this tumour should be considered when patients present with severe pain in the fingers without any obvious lesion.

\section{REFERENCES}

1. Shin DK, Kim MS, Kim SW, et al. A painful glomus tumor on the pulp of the distal phalanx. J Korean Neurosurg Soc 2010;48:185-7.

2. Kim DH. Glomus tumor of the finger tip and MRI appearance. Iowa Orthop J 1999;19:136-8.

3. Samaniego E, Crespo A, Sanz A. Key diagnostic features and treatment of subungual glomus tumor. Actas Dermosifiliogr 2009; 100:875-82.

4. Lin $\mathrm{YC}, \mathrm{Hsiao} \mathrm{PF}, \mathrm{Wu} \mathrm{YH}$, et al. Recurrent digital glomus tumor: analysis of 75 cases. Dermatol Surg 2010;36:1396-400.

5. Netscher DT, Aburto J, Koepplinger M. Subungual glomus tumor.J Hand Surg Am 2012;37:821-3.

6. Takemura N, Fujii N, Tanaka T. Subungual glomus tumor diagnosis based on imaging. J Dermatol 2006;33:389-93.

7. Glazebrook KN, Laundre BJ, Schiefer TK, et al. Imaging 
features of glomus tumors. Skeletal Radiol 2011;40:855-62.

8. Tuncali D, Yilmaz AC, Terzioglu A, et al. Multiple occurrences of different histologic types of the glomus tumor. J Hand Surg Am 2005;30:161-4.

9. Anakwe RE, McEachan JE. A glomus tumour beneath the painful unpolished nail. CMAJ 2010;182:1329.

10. Carroll RE, Berman AT. Glomus tumors of the hand: review of the literature and report on twenty-eight cases. J Bone Joint Surg Am 1972;54:691-703.

11. Matsunaga A, Ochiai T, Abe I, et al. Subungual glomus tumour: evaluation of ultrasound imaging in preoperative as- sessment. Eur J Dermatol 2007; 17:67-9.

12. Lee IJ, Park DH, Park MC, et al. Subungual glomus tumours of the hand: diagnosis and outcome of the transungual approach. J Hand Surg Eur Vol 2009;34:685-8.

13. Al-Qattan MM, Al-Namla A, Al-Thunayan A, et al. Magnetic resonance imaging in the diagnosis of glomus tumours of the hand.J Hand Surg Br 2005;30:535-40.

14. Kuru I, Oktar SO, Maralcan G, et al. Familial glomus tumor encountered in the same finger and localization in four family members. Acta Orthop Traumatol Turc 2005;39:365-8. 